\title{
Masern-Endemie in Deutschland
}

Die WHO möchte die Masern ausrotten. Um dieses Ziel zu erreichen, müssten $95 \%$ der Kinder zwei Dosen einer MMR-Vakzine erhalten. In Deutschland liegt die Durchimpfungsrate bei $\mathbf{8 9 \%}$. Aber selbst in Regionen mit überdurchschnittlichen Durchimpfungsraten kann es noch zu Masernendemien kommen, wie das Beispiel Essen (Durchimpfungsrate $92 \%$ ) zeigt.

- Deutsche Forscher beschrieben fünf Masernfälle bei Schulkindern, die alle in eine Waldorfschule in Essen gingen. Die Erkrankungen wurden am 15. März 2010 diagnostiziert. Vier Tage später wurden bereits 71 Masernfälle (68 Kinder und drei Erwachsene) registriert. Davon waren 30 Waldorfschul- kinder oder Geschwister solcher Personen. Von den 71 Fällen hatte nur ein Kind zwei MMR-Impfungen erhalten.

Serologisch war das Virus dem sehr ähnlich, das bereits im gleichen Jahr in einer Berliner Waldorfschule zu einer Masernendemie geführt hatte. Dort war es zu 62 dokumentierten Erkrankungsfällen gekommen.

\section{Kommentar}

Waldorfschulen sind anthroposophisch geführte Schulen. Anthroposophen lehnen das Impfen $a b$, und anthroposophische Ärzte sind der Auffassung, dass Infektionskrankheiten wie Masern wichtige „Meilensteine“ in der Entwicklung eines Kindes seien. Die Kinder in Waldorfschulen bleiben daher häufig ungeimpft.
So kommt es, dass sich Masernendemien ziemlich regelmäßig in solchen Institutionen ereignen.

Der letzte große Ausbruch von Masern in Deutschland war 2006. Damals erkrankten 2300 Personen, 414 davon so schwer, dass sie ins Krankenhaus mussten. Zwei Patienten starben an Enzephalitis. Vor diesem Hintergrund klingt das Wort „Meilenstein“, wie ich finde, reichlich zynisch.

E. ERNST :

\section{- H. Roggendorf et al.}

Spotlight on measles 2010: measles outbreak in a mainly unvaccinated community in Essen, Germany, March-June 2010. Euro Surveill. 2010 Jul 1;15(26). pii: 19605 